\title{
Cholera outbreaks continue
}

Background and epidemiology:

On Feb. 18, 2004, in between updates on SARS and Avian influenza, the World Health Organization reported recent outbreaks of cholera in 6 countries (Burundi, Cameroon, Mali, Mozambique, South Africa and Zambia) involving more than 14000 cases and resulting in 297 deaths. These outbreaks remind us that, among the sensational and emerging infectious diseases, old enemies such as cholera continue to plague poor communities in Africa, Asia and South America.

Since the early 19th century "Asiatic cholera" has been endemic in South Asia, especially in the Ganges Delta of India. The first pandemic occurred in 1817 , when the disease spread out of India along trade routes west as far as Russia. By the 1830s cholera had reached European cities, including London. It was in 1854 that John Snow came up with the landmark epidemiologic association between cholera and contaminated drinking-water supplies in London. This was followed by Koch's isolation of the pathogen Vibrio cholerae at the end of the 19th century.

There have been 7 pandemics of cholera and extensive epidemics, all caused by the $\mathrm{O} 1$ serotype called El Tor. The seventh pandemic originated in In-

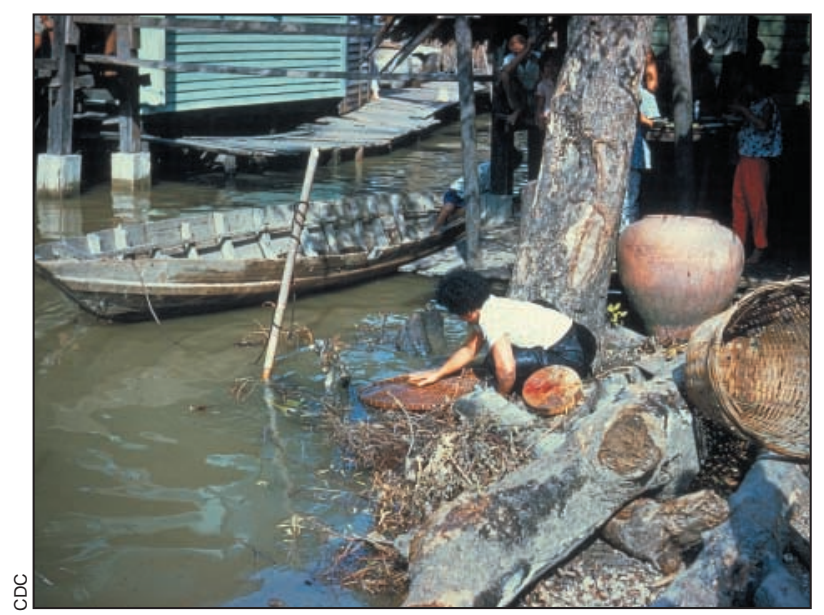

Cholera continues to plague communities around the world where clean drinking water is in short supply. donesia rather than the Ganges Delta and affected most regions of the world (India, Africa, South America and southern Europe). In 1992, a new serotype, O139, was identified in cholera outbreaks in India and Bangladesh. It afflicted more than 100000 people and resulted in more than 1400 deaths. The eighth pandemic will probably be caused by the O139 serotype.

Over the same period, substantial advances in the scientific understanding of $V$. cholerae have occurred, including classification of strains and biotypes, complete genomic sequencing of a representative isolate, characterization of the principal virulence genes, elaboration of the ecology and infection cycle of $V$. cholerae, and development and mass administration of an oral vaccine. ${ }^{1,2}$

$V$. cholerae is a motile, gramnegative curved rod that belongs to the family Vibrionaceae. It is a facultative human pathogen that exists as a natural inhabitant of surface water and multiplies and survives in association with zooplankton and phytoplankton quite independent of infected individuals. Cholera can spread both through fecal contamination of food and water by humans and through independent propagation of the pathogen in the environment. Of the more than 200 recognized O serogroups, only $\mathrm{O} 1$ and the newly emerged O139 have been associated with severe disease and cholera pandemics. These 2 strains appear to be equipped with genes that encode products that permit effective negotiation through all stages of the infection cycle, including survival in gastric $\mathrm{pH}$ levels, penetration of mucus gel in the duodenum, adherence to microvilli, colonization and propagation in the ileum, expression of cholera toxin, expulsion through diarrhea and survival in aquatic environments.
Determination of the genome sequence of $V$. cholerae $\mathrm{O} 1$ has enabled the location of virulence, regulatory and structural genes and has permitted insights into vaccine development and the relation between environmental and clinical (human) strains and climate change. There is increasing evidence that virulence gene regulation is influenced by environmental factors. The switching on of such genes is the result of a series of horizontal gene transfers that allows a benign marine bacterium to rapidly evolve into a dangerous pathogen in response to environmental cues. That is, cholera toxin genes have been shown to be located within a lysogenic bacteriophage, and propagation of this bacteriophage is influenced by environmental factors such as temperature, sunlight and salinity. ${ }^{1,2}$

Clinical management: Symptoms of cholera may vary from mild, watery diarrhea to an acute diarrhea with characteristic rice water stools. Onset of illness is usually sudden, with incubation periods varying from 6 hours to 5 days. Symptoms can rapidly progress from diarrhea, abdominal cramps, nausea and vomiting, to dehydration, shock and death. Cholera can be confirmed through culture of stool samples and serotyping the isolate, but the diagnosis remains primarily clinical. Treatment with rapid and appropriate rehydration and electrolyte replacement, using isotonic intravenous fluid therapy or oral rehydration fluid therapy, is critical because most deaths occur within the first 24 hours. Use of the "cholera cot" allows accurate recording of fluid losses. Therapy with an antimicrobial agent (erythromycin, azithromycin, ciprofloxacin, doxycycline, chloramphenicol, furazolidone or cotriamoxazole) for 1-3 days reduces the severity and duration of symptoms, although resistance to 
these agents has been increasing. ${ }^{1}$ Mild disease does not require the use of antimicrobial therapy.

Prevention: Two oral cholera vaccines are available: an attenuated live vaccine based on the genetically modified $V$. cholerae O1 strain (Orochol) given as a single dose, and a killed wholecell $V$. cholerae $\mathrm{O} 1$ strain with purified cholera toxin (Dukoral) that provides strong protection with 2 doses given 1-6 weeks apart. ${ }^{4}$ Orochol is available in Canada but is not recommended for routine use in travellers to cholera-endemic areas unless they are at high risk of exposure (e.g., health care professionals working in disease-endemic ar- eas or aid workers in refugee camps). Booster doses are needed because of the lack of long-term immunity. Travellers not at high risk are advised to exercise general food and water precautions (www.hc-sc.gc.ca /pphb-dgspsp/tmp-pmv).

Vaccination and sanitation programs should work as complementary prevention strategies in cholera-endemic areas. ${ }^{3}$ However, the protectivity of the cholera vaccines on a mass scale still needs to be defined. The Dukoral vaccine is currently being administered through a mass vaccination demonstration project in the Mozambique town of Beira. The aim is to decrease the disease's incidence in highrisk populations. The first re- sults of this project will be obtained in a year. ${ }^{4}$

\section{Erica Weir}

CMAF

Shariq Haider

Division of Infectious Diseases

McMaster University Medical Centre

Hamilton, Ont.

\section{References}

1. Shears P. Recent developments in cholera. Curr Opin Infect Dis 2001;14: 553-8.

2. Reidl J, Klose K. Vibrio cholerae and cholera: out of the water and into the host. FEMS Microbiol Rev 2002;26: 125-39.

3. Sack AD, Sack B, Balakrish Nair G, Siddiqui AK. Cholera. Seminar. Lancet 2004;363:225-36.

4. World Health Organization. Mozambique mass campaign tests the theory. Geneva: The Organization; 2004 Jan 14. Available: www.who.int/mediacentre /releases/2004/pr3/en/ (accessed 2004 Feb 23). 